\title{
Filigrane
}

Écoutes psychothérapiques

\section{Le témoin}

\section{Réal Laperrière}

Volume 19, numéro 2, automne 2010

URI : https://id.erudit.org/iderudit/1000459ar

DOI : https://doi.org/10.7202/1000459ar

Aller au sommaire du numéro

Éditeur(s)

Revue Santé mentale au Québec

\section{ISSN}

1192-1412 (imprimé)

1911-4656 (numérique)

Découvrir la revue

\section{Citer cet article}

Laperrière, R. (2010). Le témoin. Filigrane, 19(2), 75-80. https://doi.org/10.7202/1000459ar

\section{Résumé de l'article}

Inspiré par Ferenczi et sa conception du traumatisme, l'auteur propose d'explorer à travers deux courtes vignettes cliniques, une position transférentielle particulière dans laquelle l'analyste est amené à prendre la place d'un témoin, et favoriser l'inscription psychique et dans le temps de ce qui ne se présente que dans l'actuel.
Ce document est protégé par la loi sur le droit d'auteur. L'utilisation des services d'Érudit (y compris la reproduction) est assujettie à sa politique d'utilisation que vous pouvez consulter en ligne.

https://apropos.erudit.org/fr/usagers/politique-dutilisation/
Cet article est diffusé et préservé par Érudit.

Érudit est un consortium interuniversitaire sans but lucratif composé de l’Université de Montréal, l'Université Laval et l'Université du Québec à Montréal. Il a pour mission la promotion et la valorisation de la recherche. https://www.erudit.org/fr/ 


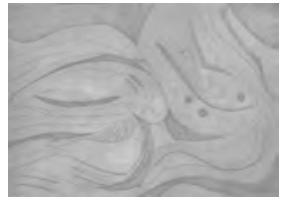

\section{Le témoin ${ }^{1}$}

\section{Réal Laperrière}

Inspiré par Ferenczi et sa conception du traumatisme, l'auteur propose d'explorer à travers deux courtes vignettes cliniques, une position transférentielle particulière dans laquelle l'analyste est amené à prendre la place d'un témoin, et favoriser l'inscription psychique et dans le temps de ce qui ne se présente que dans l'actuel.

Dans le Journal clinique Ferenczi écrit:

Ainsi, on a le choix : prendre vraiment au sérieux le rôle dans lequel on se met en tant qu'observateur bienveillant et secourable, c'est-à-dire qu'on est en fin de compte transporté avec le patient dans cette période de son passé (une façon de faire interdite contre laquelle Freud m'avait mis en garde), avec, pour résultat, que nousmêmes comme le patient croyons en cette réalité, c'est-à-dire en une réalité existante dans le présent et non momentanément transposée dans le passé. (Ferenczi, 1932, 71).

Il semble donc convier l'analyste à adopter un « rôle», c'est-à-dire une position transférentielle particulière face à des expériences catastrophiques dans lesquelles l'affect, devenu indice de réalité (Reid, 2010), amène en séance, ici-et-maintenant, la présentation d'un vécu traumatique. Présentation et non pas représentation dont l'inscription psychique pourra être favorisée par cette position prise par l'analyste.

Julie

Au cours des premières années de sa psychothérapie, Julie, une femme dans la cinquantaine, me demandait sans cesse, et avec une insistance chargée d'angoisse: "Mais vous, est-ce que vous me croyez?» Ne s'interrogeant pas elle-même sur ce qui pouvait l'amener à formuler répétitivement cette question, elle n'en appelait qu'à une réponse immédiate... à laquelle elle ne pourrait jamais suffisamment croire. Est-ce que je pouvais croire, en effet, cette incroyable série de traumatismes et de réactions de survie qui semblaient avoir marqué son parcours de vie depuis la toute petite enfance, et dont elle disait n'avoir jamais parlé, tant pour se protéger de la honte intolérable d'avoir été une « victime» (mot qu'elle arrivait à peine à prononcer tellement il lui faisait horreur), que de la culpabilité, rempart ultime contre l'impuissance, d'avoir eu recours à différents moyens pour tenter de s'en sortir. Son mari, ses enfants, même ses meilleures amies ne savaient que peu de choses à son sujet: la mort de sa mère lorsqu'elle était âgée de trois ans, son placement dans une famille d'accueil, la fuite de 
cette famille le jour de sa majorité et la rupture définitive avec celle-ci. Mais pour le reste, tout était resté secret: l'abandon par son père au moment de son placement, la perte brutale de tout contact avec ses frères et sœurs, les mauvais traitements infligés par une mère d'accueil folle, qui «pétait sa coche» de façon imprévisible et dont elle garde des séquelles dans son corps (fractures mal soignées) encore aujourd'hui, ses rapports sexuels, dès l'âge de 12 ans, avec un homme dans la vingtaine, ses nombreuses fugues, ses années de prostitution de 14 à 17 ans, au cours desquelles elle se mit quelques fois gravement en danger lors de voyages à New York et Toronto, la violence inouïe d'une agression par un couple pervers, en qui elle avait d'abord mis ses espoirs et sa confiance. «Est-ce que vous me croyez?» insistait-elle avec un désarroi dans la voix, laissant entendre que le moindre doute à ce sujet serait vécu comme une catastrophe, répétant le désaveu par les adultes de la détresse éprouvée dans son enfance, désaveu auquel elle s'est depuis longtemps identifiée et qui lui a permis, jusqu'au moment de crise l'ayant amenée en psychothérapie, de «fonctionner» dans la vie, mais en ne se croyant pas elle-même: «Non, je n'ai pas subi cela, je l'ai provoqué... c'est de ma faute... je gardais toujours le contrôle. » Bien sûr que je la croyais sur parole... en pensant qu'on n'a pas à demander à un enfant s'il a trouvé ou créé son objet transitionnel et que l'analysant comme l'analyste doivent pour un temps «se soustraire au dilemme de l'imaginé et du vécu, de la remémoration et de la fantasmatisation » (Donnet, 1995, 68). Mais aucune intervention de ma part ne semblait lui convenir, et encore moins l'absence d'intervention. «Me croyez-vous vraiment? » continuait-elle de me demander régulièrement, jusqu'à ce que, en fin de parcours analytique, elle me fasse comprendre que c'est autre chose qu'elle avait attendu de moi toutes ces années. Ainsi un jour, se disant satisfaite du travail accompli avec moi et se sentant depuis un bon moment, vivante, en contact avec elle-même et avec les autres, elle se demande de quelle façon elle pourra bien arriver à me quitter pour poursuivre sa route sans nos séances hebdomadaires. Survient alors un rêve: elle se promène dans la rue de son enfance, et est invitée à entrer dans la maison qu'elle habitait. A l'extérieur, cette maison n'a pas changé depuis 45 ans. Mais à l'intérieur, elle a été complètement refaite, par la femme qui y habite maintenant. Elle se promène, admirative des murs faits de terre et de racines, merveilleusement vernis, et des nouvelles pièces, vastes et lumineuses, qui ont été ajoutées à la maison. Elle se dit alors qu'elle aimerait bien que quelqu'un soit là, à ses côtés, pour partager sa découverte et s'émerveiller avec elle.

Ayant terminé le récit de son rêve, souriante, elle me dit avoir compris ce qu'elle attendait de moi: que je puisse être pour elle un témoin, non pas de ce qui s'était passé autrefois («Me croyez-vous?»), puisqu'alors je n’y étais pas. Mais un témoin de ce qu'elle vivait avec moi à chaque séance. «Et je sais maintenant que je pourrai partir d'ici quand nous aurons revisité le chemin parcouru, et que je saurai que vous êtes témoin de mon voyage... ». Ce n'est qu'après coup que j'ai pensé que j'avais dû jouer, à mon insu, ce rôle de témoin, et que cela l'avait peut être aidée, comme en témoignent apparemment le rêve et le questionnement sur celui-ci, à inscrire psychiquement et à représenter ce qui ne pouvait pendant longtemps que se présenter dans l'actuel d'une séance. A instaurer, donc, une temporalité. 


\section{Catherine}

Lors d'une hospitalisation à la suite d'une première tentative de suicide et après une évaluation psychologique, Catherine, 13 ans, demande à faire une psychothérapie avec moi. Derrière un jeu de séduction manifeste elle demeure très à distance en séance et ne parait pas s'engager dans la démarche thérapeutique à laquelle elle décide de mettre un terme après une douzaine de rencontres. Elle obtient ensuite son congé de l'hôpital. Peu de temps après, elle est réadmise en urgence: des policiers l'ont arrêtée sur le pont Jacques-Cartier alors qu'elle menaçait de sauter. Elle demande à me revoir, et nous ré-instaurons des séances régulières qu'elle a beaucoup de difficulté à tolérer : elle apparait très angoissée, dit n'avoir rien à dire, veut partir après seulement quelques minutes... tout en continuant à revenir me voir de façon assidue. Jusqu'à ce qu'un événement vienne changer la donne entre nous.

Je marche dans un corridor de l'hôpital en route vers mon bureau et je croise Catherine accompagnée de son infirmière. Elle fait comme si elle ne me voyait pas continuant à parler joyeusement avec son accompagnatrice. Je poursuis mon chemin pensant que j'ai rendez-vous avec elle pour sa séance dans une quarantaine de minutes. Puis, un peu plus loin, j'entends hurler «Catherine!». Je me retourne et je vois alors celle-ci suspendue dans le vide de la cage d'escalier (nous sommes au $4^{e}$ étage) et retenue par les mains par son infirmière qui appelle maintenant à l'aide. J'accours et je saisis moi aussi Catherine qui ne bouge pas et reste en suspens au-dessus du vide, jusqu'à l'arrivée des agents de sécurité. Le regard absent, elle se laisse mettre sur la civière et ramener à son unité, tandis que son infirmière en pleurs, et moi, sidéré, sommes sous le choc. J'ai saisi Catherine, et je suis saisi par elle.

Dans les séances qui suivent cet incident, Catherine est très différente avec moi: elle insiste pour que je lui raconte en détail ce que j'ai vu, me faisant reprendre plusieurs fois la séquence des événements. Pourquoi donc cette répétition qui me semble révéler tout autant une demande, une aspiration à la symbolisation (Donnet, 1995) qu'un refus d'inscription, à travers ce qui pourrait être donné à voir de mon propre psychisme? En effet elle parle peu, mais, très présente, elle m'écoute avec attention en tant que témoin, et non interprète, de ce qui s'est passé.

Au rythme de deux séances hebdomadaires, je verrai Catherine jusqu'à ses 18 ans. Au cours de ce voyage très accidenté, elle fera souvent de moi, par ses agirs parfois spectaculaires et de plus en plus directement reliés à notre relation, un témoin, tout autant qu'un acteur dans un scénario en voie d'élaboration.

Rappelons-nous que pour Ferenczi, le traumatisme résulte:

- des mouvements passionnels des adultes;

- des désaveux de ces mêmes adultes quant à la souffrance psychique, notamment celle de l'enfant, désaveux pouvant être vécus comme un «terrorisme de la souffrance» et avoir pour conséquence une entrave dans son autonomie de pensée; 
- de l'introjection du sentiment inconscient de culpabilité de l'adulte.

"Que se passe-t-il, se demande Ferenczi, quand la souffrance augmente et dépasse la force de compréhension du petit être? L'usage commun caractérise ce qui s'ensuit par l'expression : l'enfant est hors de lui.» $(1932,79)$. L'agressé se retire de luimême pour devenir témoin extérieur de l'expérience traumatique.

Avec Julie et Catherine, qui ont toutes deux vécu de graves traumatismes pendant l'enfance et qui présentent certainement un fonctionnement traumatique de la psyché (Reid, 2008), il m’a semblé qu'un des courants forts du rapport transférocontre-transférentiel a été l'assignation de l'analyste comme témoin. Mais témoin de quoi, et pour quoi?

Le patient doit, avec l'analyste, répéter le traumatisme, nous dit Monique Schneider, «non pour recommencer en se soumettant à la loi de l'identique, mais pour faire advenir ce qui n'est pas encore advenu» $(1988,219)$. Ce qui n'est pas advenu, c'est une expérience ayant du sens, c'est-à-dire comportant « les trois sens du mot sens» (DaSilva, 2010) : le sens comme sensation, le sens comme sentiment, le sens comme signification. L'analyste est donc d'abord amené à occuper la place du patient identifié au désaveu, à incarner sa partie «hors de lui » c'est-à-dire clivée, morte et froidement observatrice, et ainsi à « reproduire de ses mains le meurtre perpétré jadis sur le patient» (Ferenczi, 1932, 103). Par le jeu du transfert, il devient donc celui qui désavoue la souffrance psychique de l'enfant en détresse, celui qui sait mais fait comme si de rien n'était, témoin extérieur impuissant à secourir. Par ses erreurs techniques et ses manquements auprès du patient, il incarne les défaillances précoces de l'environnement (Press, 2008). C'est ainsi que Julie, impossible à réassurer, était profondément convaincue que je ne la croyais pas. Et que Catherine, une fois bien engagée dans sa psychothérapie, me disait souvent: «Tu ne sens rien, tu n'es pas un humain, tu n'es qu'un psychologue.»

Or, c'est au cœur de cette reprise du trauma dans l'actuel que l'analysant lance aussi un "un appel désespéré: un tiers est convoqué d'urgence» (Schneider, idem, 22). Ce tiers-témoin peut alors devenir celui qui témoigne en éprouvant, et cautionne l'éprouvé de l'enfant. Ainsi Catherine me faisant sentir par ses agirs suicidaires répétés et ses menaces de s'engager dans la prostitution, la détresse et l'impuissance de l'enfant confronté à une mère dépressive, suicidaire et insensible aux agressions sexuelles subies par sa toute petite fille: «Ne t'en fais pas, j'ai connu pire! » lui disaitelle. Julie, de son côté, me faisant éprouver ce qu'il en est de ne pas être cru et de voir ainsi sa propre parole disqualifiée. L'analysant se met alors lui-même en position de témoin du contre-transfert de l'analyste, et de sa capacité à transformer cette quantité d'excitation en qualité, par le travail de la pensée et de la requalification de l'affect.

Pour Ferenczi, le travail de l'analyste consiste à réanimer la partie clivée et morte du patient, en arrivant à penser pour lui et avec lui l'événement traumatique. «Penser le traumatisme» n'a que peu à voir avec les faits de l'histoire, si fascinants et séduisants soient-ils par ce qu'ils donnent à voir ainsi que par leur potentiel explicatif (comme dans les récits spectaculaires de Julie et de Catherine). Il s'agit plutôt du fonctionnement traumatique de la psyché (Reid, 2008) - qui bien sûr ne peut être 
sans un certain rapport de causalité avec les faits historiques - tel qu'il se manifeste dans l'ici-et-maintenant de la séance, c'est-à-dire ayant l'hallucinatoire comme principe organisateur, ce qui entraîne une indifférenciation du dedans et du dehors ainsi qu'une équivalence représentation-perception empêchant toute élaboration psychique du traumatisme (Reid, 2010). L'analyste qui écoute, nous dit Schneider (1992) est tout autant témoin que complice. Il est celui qui donne existence à une scène, et qui témoigne du fait qu'il y a là quelque chose à percevoir (je dirais «à éprouver») et à penser, plutôt qu'à répéter. Il actualise donc une menace puisque pour le sujet, on ne peut survivre à la scène qu'en cessant justement de percevoir et de penser. C'est l'opération de pensée proposée par l'analyste qui provoque le trauma appréhendé et le débordement.

Ce débordement, lorsqu'il rencontre l'offre d'une présence-écoute par un analyste, va amener le patient à convier celui-ci à être porteur de la partie de lui-même qui, par identification à l'agresseur et à sa culpabilité s'est faite d'abord parent de son parent puis parent de lui-même: «Sauve-moi!», «Emmène-moi loin!» m’implorait Catherine. Mais il devient aussi un appel pressant à une possible transformation : faire du «psychique» avec ce qui ne cesse de se présenter comme du pulsionnel. Encore faut-il, nous dit Mauger (1995) que l'analysant accepte de perdre son traumatisme, car faire exister la chose traumatique c'est la faire mourir tout autant. Arriver à faire de cet hallucinatoire, par la médiation du travail de l'analyste, une expérience de transitionnalité dans laquelle peut être toléré un indécidable (dedans ou dehors? sujet ou objet?) qui ouvre la voie à l'élaboration, voilà tout le défi de la cure.

Peut-on dire qu'un chemin peut alors se tracer de la sensation à la représentation, puis à l'érotisation, et que l'analysant peut en arriver à devenir témoin de lui-même par la mise à jour ou la construction de fantasmes régulateurs et organisateurs? Ainsi, pour Catherine, celui qui va progressivement émerger au fil des séances comme «sous-entendu» de ses agirs tranférentiels: le fantasme d'être en train de tomber dans le vide mais attrapée juste au bon moment et retenue par moi, suspendue, arrêtée dans le temps et l'espace. Pour Julie, celui apparaissant dans son rêve de fin de parcours: pouvoir enfin rentrer à la maison (celle de son enfance), se réapproprier son histoire (les murs faits de racines), sortir de la répétition (la maison qui extérieurement n'a pas changé depuis 45 ans mais qui intérieurement a été rénovée par la femme qui y habite) pour pouvoir vivre du nouveau (les nouvelles pièces vastes et lumineuses), auprès cette fois d'un témoin, objet présent-absent en elle, pouvant ressentir avec elle (le désir de quelqu'un à ses côtés). Faire ainsi de l'histoire de la construction, expérience transitionnelle de la cure, une pièce essentielle à la construction de son histoire (Donnet, 1995).

En arpentage, le témoin est un piquet servant à marquer un emplacement; en archéologie, il s'agit de la hauteur laissée intacte au cours de fouilles; en peinture, c'est l'espace non nettoyé après la restauration d'un tableau; en sport, c'est le bâtonnet que se passent les coureurs à relais (Petit Robert). Le témoin a donc toujours à voir avec le mouvement dans le temps et l'espace. Ainsi, l'analyste-témoin a-t-il à 
servir à un moment donné de la cure, de marqueur du mouvement ré-instauré dans le psychisme, qui permet de s'extraire de l'actuel et du destin («pur présent appréhendé comme ayant la pesanteur de l'éternité » Schneider, 1992, 78) pour accéder au temps et à la destinée (Bollas, 1989).

Réal Laperrière 1209 Fleury Est, app. A Montréal, Québec, H2C 1R2 laperrierereal@videotron.ca

\section{Notes}

1. Version modifiée d'un texte présenté à la Société psychanalytique de Montréal, le 17 décembre 2009.

\section{Références}

BOLLAS, C., 1989, Les forces de la destinée, Calmann-Levy, Paris.

DaSILVA, G., 2010, Communication personnelle.

DONNET, J.-L., 1995, Le divan bien tempéré, Presses universitaires de France, Paris.

FERENCZI, S., 1932, Journal clinique, Payot, 1985.

MAUGER, J., 1995, Ne voyez-vous pas ce que je veux dire? Trans, été, 9-25.

PRESS, J., 2008, Construction avec fin, sans fin, Revue française de psychanalyse, 72, 5, 1269-1337.

REID, W., 2008, Un nouveau regard sur la pulsion, le trauma et la méthode analytique. Première partie: une théorie de la psyché, Filigrane, 17, 1, 68-94.

REID, W., 2010, Les avatars du trauma structurel ou les voies psychiques de l'accomplissement du désir, Revue française de psychanalyse, 74, 1, 51-69.

SCHNEIDER, M., 1988, Le trauma et la filiation paradoxale. De Freud à Ferenczi, Ramsay.

SCHNEIDER, M., 1992, La part de l'ombre. Approche d'un trauma au féminin, Aubier. 